\title{
THE REDUCTION NUMBER OF AN IDEAL AND THE LOCAL COHOMOLOGY OF THE ASSOCIATED GRADED RING
}

\author{
THOMAS MARLEY
}

(Communicated by Louis J. Ratliff, Jr.)

\begin{abstract}
Let $(R, m)$ be a local ring and $I$ an $m$-primary ideal. A result of Trung shows that if the local cohomology of $g r_{I}(R)$ satisfies certain conditions, then the reduction number of $I$ is independent of the minimal reduction chosen. These conditions consist of $t=\operatorname{dim} R-\operatorname{grade} g r_{I}(R)^{+}$inequalities. We show that if $R$ is Cohen-Macaulay, then one of these inequalities is always satisied, while another can often be easily checked. Applications are then given in two-dimensional Cohen-Macaulay rings. For instance, we show that if the Hilbert function of $I$ equals the Hilbert polynomial of $I$ for all integers greater than 1 , then the reduction number is independent of the choice of minimal reduction.
\end{abstract}

\section{INTRODUCTION}

Let $(R, m)$ be a $d$-dimensional commutative Noetherian local ring and $I$ an $m$-primary ideal. We define the Hilbert function of $I$ as follows: for $n \geq 1$ we set $H_{I}(n)=\lambda\left(R / I^{n}\right)$ (where $\lambda$ denotes length), and for $n \leq 0$ we set $H_{I}(n)=0$. It is well known that this function coincides with a polynomial $P_{I}(n)$ of degree $d$ for all sufficiently large integers $n$. With this in mind, we let

$$
n(I)=\max \left\{n \in \mathbf{Z} \mid P_{I}(n) \neq H_{I}(n)\right\} .
$$

Note that $H_{I}(n)$ and $P_{I}(n)$ are defined for all integers $n$, so $n(I)$ can be, and often is, negative. Ooishi [O] refers to $n(I)$ as the postulation number of $I$ and we adopt that term here.

Throughout this paper we will always assume the residue field $R / m$ is infinite. This being the case, we define a minimal reduction of $I$ to be an ideal $(\underline{x})=$ $\left(x_{1}, \ldots, x_{d}\right) \subset I$ such that $(\underline{x}) I^{n}=I^{n+1}$ for some nonnegative integer $n$ (see [NR]). For convenience, let $M R(I)$ denote the set of minimal reductions of $I$. For $J \in M R(I)$, we define $r_{J}(I)$ to be $\min \left\{n \in \mathbf{Z} \mid J I^{n}=I^{n+1}\right\}$. Also, we define $r(I)$, the reduction number of $I$, to be $\min \left\{r_{J}(I) \mid J \in M R(I)\right\}$.

In this paper, we will consider some conditions on $I$ that force $r_{J}(I)$ to be independent of the choice of minimal reduction. Huckaba $[\mathrm{H}]$ and Trung $[\mathrm{T}]$

Received by the editors January 29, 1991 and, in revised form, June 17, 1991; the contents of this paper were presented at the 861st meeting of the AMS in Denton, TX, November 3, 1990.

1991 Mathematics Subject Classification. Primary 13D03; Secondary 13H15.

Key words and phrases. Local cohomology, reduction, Hilbert function. 
independently proved that if grade $g r_{I}(R)^{+} \geq d-1$ then $r_{J}(I)$ is independent of $J$. Furthermore, it is proved in [S, M] that if $R$ is Cohen-Macaulay (CM, for short) and grade $g r_{I}(R)^{+} \geq d-1$, then $r_{J}(I)=n(I)+d$ for all $J \in$ $M R(I)$. We give another proof of this fact (Corollary 2.2) by making use of some observations made by Trung in [T]. Perhaps just as important, however, we uncover some interesting information on the local cohomology of $g r_{I}(R)$ that sets these rings apart from arbitrary graded rings generated by 1 -forms. This information (Theorem 2.1) is particularly useful in providing some insight on the independence of the reduction number when $d=2$ and grade $g r_{I}(R)^{+}=0$ (see §3). More applications in dimension two will be forthcoming in [HM].

\section{Preliminaries}

Let $S=\bigoplus_{n>0} S_{n}$ be a Noetherian graded ring where $S_{0}$ is an Artinian local ring and $S$ is generated by 1 -forms over $S_{0}$. If $M=\bigoplus_{n \in \mathbf{Z}} M_{n}$ is a finitely generated graded $S$-module, we let $H_{M}(n)=\lambda_{S_{0}}\left(M_{n}\right)$. Then there is a polynomial $P_{M}(n)$ of degree $\operatorname{dim} M-1$ such that $P_{M}(n)=H_{M}(n)$ for $n$ sufficiently large. Note that if $(R, m)$ is a local ring, $I$ is an $m$-primary ideal, and $G=g r_{I}(R)$, then

$$
P_{G}(n)-H_{G}(n)=\Delta^{1}\left(P_{I}(n)-H_{I}(n)\right),
$$

where $\Delta^{1}(f(n))=f(n+1)-f(n)$ for any integer-valued function $f$.

For $i \geq 0$, we let $H_{S^{+}}^{i}(M)=\bigoplus_{n \in \mathbf{Z}} H_{S^{+}}^{i}(M)_{n}$ be the $i$ th local cohomology module of $M$ with support in $S^{+}$. Serre showed that these modules are Artinian and that each $H_{S^{+}}^{i}(M)_{n}$ is finitely generated. Thus, $H_{S^{+}}^{i}(M)_{n}=0$ for $n$ sufficiently large. If $H_{S^{+}}^{i}(M) \neq 0$, we let

$$
a_{i}(M)=\max \left\{n \in \mathbf{Z} \mid H_{S^{+}}^{i}(M)_{n} \neq 0\right\} .
$$

For convenience, we define $a_{i}(M)=-\infty$ for $i<\operatorname{depth}_{S^{+}} M$ and $a_{i}(M)=\infty$ for $i>\operatorname{dim} M$. We will use $a_{i}(I)$ to denote $a_{i}\left(g r_{I}(R)\right)$.

An element $g \in S_{1}$ is called superficial for $M$ if $\left(0:_{M} g\right)_{n}=0$ for all $n$ sufficiently large. If $S_{0}$ has an infinite residue field then superficial elements for $M$ always exist [ZS, Chapter VIII]. A sequence of elements $g_{1}, \ldots, g_{t}$ is called a superficial sequence for $M$ if $g_{i+1}$ is superficial for $M /\left(g_{1}, \ldots, g_{i}\right) M$ for $0 \leq i \leq t-1$. It will be crucial in the proof of Theorem 2.1 to understand how the invariants $a_{i}(I)$ behave with respect to superficial elements. This relationship was illuminated by Trung in the following lemma. Because of its importance in our arguments, we supply the proof along with the statement.

Lemma 1.1 [T, Lemma 2.3]. Let $S$ and $M$ be as above and let $g \in S_{1}$ be superficial for $M$. Then for $i \geq 0$,

$$
a_{i+1}(M)+1 \leq a_{i}(M / g M) \leq \max \left\{a_{i}(M), a_{i+1}(M)+1\right\} .
$$

Proof. First note that $H_{S^{+}}^{0}\left(\left(0:_{M} g\right)\right)=\left(0:_{M} g\right)$ and $H_{S^{+}}^{i}\left(\left(0:_{M} g\right)\right)=0$ for $i \geq 1$. Then from the exact sequence

$$
0 \rightarrow\left(0:_{M} g\right) \rightarrow M \rightarrow M /\left(0:_{M} g\right) \rightarrow 0,
$$

we see that $H_{S^{+}}^{i}(M) \cong H_{S^{+}}^{i}\left(M /\left(0:_{M} g\right)\right)$ for $i \geq 1$. And from the exact sequence

$$
0 \rightarrow M /(0: M \quad g)[-1] \stackrel{g}{\rightarrow} M \rightarrow M / g M \rightarrow 0,
$$


we obtain the exact sequences

$$
H_{S^{+}}^{i}(M)_{n} \rightarrow H_{S^{+}}^{i}(M / g M)_{n} \rightarrow H_{S^{+}}^{i+1}(M)_{n-1} \rightarrow H_{S^{+}}^{i+1}(M)_{n}
$$

for each $i \geq 0$ and $n \in \mathbf{Z}$. Analyzing these sequences easily yields the desired inequalities.

With the help of this lemma, Trung was able to establish the following relationship between the reduction number and the $a_{i}(I)$ 's.

Lemma 1.2 [T, Proposition 3.2]. Let $(R, m)$ be a local ring of dimension $d$ and $I$ an $m$-primary ideal. Then for any $J \in M R(I)$,

$$
a_{d}(I)+d \leq r_{J}(I) \leq \max _{0 \leq i \leq d}\left\{a_{i}(I)+i\right\}
$$

From this we see that if $a_{i}(I) \leq a_{d}(I)+d-i$ then $r_{J}(I)=a_{d}(I)+d$ for all $J \in M R(I)$ and so $r_{J}(I)$ is independent of $J$. There are many examples of when this occurs, particularly in dimension two (see $\S 3$ of this paper as well as [HM]). The link between the local cohomology of $g r_{I}(R)$ and the postulation number of $I$ is given by the following formula first noticed by Grothendieck (cf. [EGA, Proposition 2.1.5]):

Lemma 1.3. Let $S=S_{0}\left[x_{1}, \ldots, x_{r}\right]$ be a Noetherian graded ring, where $S_{0}$ is an Artinian local ring and $\operatorname{deg} x_{i}=1$ for $1 \leq i \leq r$. Then for any finitely generated graded $S$-module $M$,

$$
P_{M}(n)-H_{M}(n)=\sum_{i=0}^{\infty}(-1)^{i+1} \lambda\left(H_{S^{+}}^{i}(M)_{n}\right)
$$

for all $n \in \mathbf{Z}$.

Proof. We proceed by induction on $r$. If $r=0$ then $P_{M}(n)=0$ and $H_{S^{+}}^{0}(M)=$ $M$ and the formula holds. Otherwise, consider the exact sequence

$$
0 \rightarrow K \rightarrow M[-1] \stackrel{x_{r}}{\rightarrow} M \rightarrow C \rightarrow 0 .
$$

Where the map between $M[-1]$ and $M$ is multiplication by $x_{r}$, while $K$ and $C$ are defined to be the kernel and cokernel, respectively. Since $x_{r} K=x_{r} C=0$, $K$ and $C$ are modules over $S / x_{r} S$. By induction, the theorem is satisfied for the modules $K$ and $C$. That is, if we let $\chi_{M}(n)$ denote the right-hand side of (\#), we have $P_{K}(n)-H_{K}(n)=\chi_{K}(n)$ and $P_{C}(n)-H_{C}(n)=\chi_{C}(n)$ for all $n \in \mathbf{Z}$. But from the exact sequence above, we have for all integers $n$

$$
\begin{aligned}
\Delta^{1}\left(P_{M}(n)-H_{M}(n)\right) & =P_{C}(n+1)-H_{C}(n+1)-P_{K}(n+1)+H_{K}(n+1) \\
& =\chi_{C}(n+1)-\chi_{K}(n+1) \\
& =\chi_{M}(n+1)-\chi_{M}(n)=\Delta^{1}\left(\chi_{M}(n)\right) .
\end{aligned}
$$

The third equality follows from the fact that $\chi$ is additive on short exact sequences. Now since both $P_{M}(n)-H_{M}(n)$ and $\chi_{M}(n)$ vanish for $n$ sufficiently large, we obtain that $P_{M}(n)-H_{M}(n)=\chi_{M}(n)$ for all $n \in \mathbf{Z}$.

Applying this lemma to the case $M=g r_{I}(R)$, we note the following

Remark 1.4. Let $(R, m)$ be a local ring and $I$ an $m$-primary ideal. Then

(a) $n(I) \leq \max \left\{a_{0}(I), \ldots, a_{d}(I)\right\}$. 
(b) If there exists $j \in\{0, \ldots, d\}$ such that $a_{i}(I)<a_{j}(I)$ for all $i \neq j$, then we have equality in (a).

(c) Combining (b) with Lemma 1.2, we see that if $a_{i}(I)<a_{d}(I)$ for all $i<d$ then $r_{J}(I)=n(I)+d$ for all $J \in M R(I)$.

\section{THE MAIN THEOREM}

A sequence of elements $x_{1}, \ldots, x_{k} \in I \backslash I^{2}$ is called a superficial sequence for $I$ if the images $x_{1}^{*}, \ldots, x_{k}^{*} \in I / I^{2}$ form a superficial sequence for $g r_{I}(R)$.

Theorem 2.1. Let $(R, m)$ be a $C M$ local ring, I an m-primary ideal, and $G=g r_{I}(R)$. Let $t=\operatorname{grade} G^{+}$. Then

(a) $a_{t}(I)<a_{t+1}(I)$.

(b) Either $a_{t+1}(I)<a_{t+2}(I)$ or

$$
a_{t+1}(I)=\max \left\{n \in \mathbf{Z} \mid\left(\left(I^{n+t+1}, x_{1}, \ldots, x_{t}\right): x_{t+1}\right) \neq\left(I^{n+t}, x_{1}, \ldots, x_{t}\right)\right\}
$$

where $x_{1}, \ldots, x_{t+1}$ is any superficial sequence for $I$.

Proof. Suppose first that $t=0$. Choose $x \in I \backslash I^{2}$ such that $x$ is superficial for $I$. Let $\bar{G}=g r_{I /(x)}(R /(x))$ and consider the exact sequence

$$
0 \rightarrow K \rightarrow G / x^{*} G \rightarrow \bar{G} \rightarrow 0
$$

where $K_{n}=\left(I^{n+1}+\left(I^{n} \cap(x)\right)\right) /\left(I^{n+1}+x I^{n-1}\right)$. Since $x$ is not a zero-divisor on $R$, we find that

$$
K_{n} \cong\left(I^{n}: x\right) /\left(I^{n-1}+\left(I^{n+1}: x\right)\right) .
$$

As $x$ is superficial for $I,\left(I^{n}: x\right)=I^{n-1}$ for $n$ sufficiently large (see [K, Lemma 4] for example), and so $K_{n}=0$ for $n$ sufficiently large. Clearly

$$
\begin{aligned}
a_{0}(K) & =\max \left\{n \in \mathbf{Z} \mid K_{n} \neq 0\right\} \\
& \leq \max \left\{n \in \mathbf{Z} \mid\left(I^{n}: x\right) \neq I^{n-1}\right\} .
\end{aligned}
$$

On the other hand, if $n>a_{0}(K)$ then by induction we have that for all $k \geq 1$

$$
\left(I^{n}: x\right)=I^{n-1}+\left(I^{n+k}: x\right) \text {. }
$$

As $\left(I^{n+k}: x\right) \subset I^{n-1}$ for $k$ sufficiently large, we see that $\left(I^{n}: x\right)=I^{n-1}$. Hence

$$
a_{0}(K)=\max \left\{n \in \mathbf{Z} \mid\left(I^{n}: x\right) \neq I^{n-1}\right\} \text {. }
$$

Now

$$
\begin{aligned}
a_{0}(I) & =\max \left\{n \in \mathbf{Z} \mid H_{G^{+}}^{0}(G)_{n} \neq 0\right\}=\max \left\{n \in \mathbf{Z} \mid\left(0: x^{*}\right)_{n} \neq 0\right\} \\
& =\max \left\{n \in \mathbf{Z} \mid\left(I^{n+2}: x\right) \cap I^{n} \neq I^{n+1}\right\} .
\end{aligned}
$$

Clearly then, $a_{0}(I) \leq a_{0}(K)-2$. But from the exact sequence $(*)$ and Lemma 1.1 , we have

$$
a_{0}(I)+2 \leq a_{0}(K) \leq a_{0}\left(G / x^{*} G\right) \leq \max \left\{a_{0}(I), a_{1}(I)+1\right\} .
$$

This implies that $a_{0}(I) \leq a_{1}(I)-1$, which proves part (a) in the case $t=0$.

For part (b), first note that

$$
a_{1}(I)+1 \leq a_{0}\left(G / x^{*} G\right) \leq \max \left\{a_{0}(I), a_{1}(I)+1\right\} .
$$


Since $a_{0}(I)<a_{1}(I)$, this implies that $a_{0}\left(G / x^{*} G\right)=a_{1}(I)+1$. Since $\operatorname{dim} K=$ $0,(*)$ induces the exact sequence

$$
0 \rightarrow H_{G^{+}}^{0}(K) \rightarrow H_{G^{+}}^{0}\left(G / x^{*} G\right) \rightarrow H_{G^{+}}^{0}(\bar{G}) \rightarrow 0 .
$$

Hence, $a_{0}\left(G / x^{*} G\right)=\max \left\{a_{0}(\bar{G}), a_{0}(K)\right\}$. We now establish the following Claim. If $a_{1}(I) \geq a_{2}(I)$ then $a_{0}(\bar{G})<a_{0}\left(G / x^{*} G\right)$.

Proof. Suppose $a_{0}(\bar{G})=a_{0}\left(G / x^{*} G\right)$. Then $\operatorname{depth}_{\bar{G}^{+}} \bar{G}=0$. Otherwise, $a_{0}(K)$ $\leq a_{0}\left(G / x^{*} G\right)=a_{0}(\bar{G})=-\infty$, which would imply that $x^{*}$ is a regular element in $G$, contradicting that $t=0$. Therefore,

$$
a_{0}\left(G / x^{*} G\right)=a_{0}(\bar{G})=a_{0}(I /(x))<a_{1}(I /(x))=a_{1}(\bar{G})=a_{1}\left(G / x^{*} G\right) .
$$

The inequality follows from the depth zero case of part (a), while the final equality is due to the fact that $\operatorname{dim} K=0$. Since $a_{0}\left(G / x^{*} G\right)=a_{1}(I)+1$, we have $a_{1}\left(G / x^{*} G\right)>a_{1}(I)+1$. But

$$
a_{2}(I)+1 \leq a_{1}\left(G / x^{*} G\right) \leq \max \left\{a_{1}(I), a_{2}(I)+1\right\} .
$$

Therefore, $a_{1}\left(G / x^{*} G\right)=a_{2}(I)+1$ and hence $a_{1}(I)<a_{2}(I)$, a contradiction. This proves the claim.

So suppose now that $a_{1}(I) \geq a_{2}(I)$. Since $a_{0}\left(G / x^{*} G\right)=\max \left\{a_{0}(K), a_{0}(\bar{G})\right\}$, the claim implies that $a_{0}\left(G / x^{*} G\right)=a_{0}(K)$. Since $a_{1}(I)=a_{0}\left(G / x^{*} G\right)-1$, we get from $(* *)$ that

$$
a_{1}(I)=a_{0}(K)-1=\max \left\{n \in \mathbf{Z} \mid\left(I^{n+1}: x\right) \neq I^{n}\right\} .
$$

This completes the case when $t=0$.

Now suppose $t>0$. Choose any superficial element $x \in I \backslash I^{2}$. Since

$$
\left(0: x^{*}\right) \subset H_{G^{+}}^{0}(G)=0,
$$

we must have that $x^{*}$ is a regular element in $G$. Hence $G / x^{*} G \cong \bar{G}$ and so, by Lemma 1.1, $a_{t-1}(I /(x))=a_{t-1}\left(G / x^{*} G\right)=a_{t}(I)+1$. By induction, $a_{t-1}(I /(x))<a_{t}(I /(x))$. Now if $a_{t}(I) \geq a_{t+1}(I)$ then

$$
\begin{aligned}
a_{t}(I /(x)) & =a_{t}\left(G / x^{*} G\right) \leq \max \left\{a_{t}(I), a_{t+1}(I)+1\right\} \\
& \leq \max \left\{a_{t}(I), a_{t}(I)+1\right\} \leq a_{t-1}(I /(x)),
\end{aligned}
$$

a contradiction. Therefore, $a_{t}(I)<a_{t+1}(I)$ and part (a) is proved.

For (b), note that part (a) coupled with Lemma 1.1 gives that $a_{t}(I /(x))=$ $a_{t+1}(I)+1$.

Now suppose $a_{t+1}(I) \geq a_{t+2}(I)$. Then

$$
\begin{aligned}
a_{t+1}(I /(x)) & \leq \max \left\{a_{t+1}(I), a_{t+2}(I)+1\right\} \\
& \leq \max \left\{a_{t+1}(I), a_{t+1}(I)+1\right\} \leq a_{t}(I /(x)) .
\end{aligned}
$$

So by induction, we have

$$
\begin{aligned}
a_{t+1}(I)= & a_{t}(I /(x))-1 \\
= & \max \left\{n \in \mathbf{Z} \mid\left(\left(I^{n+t}, x, x_{2}, \ldots, x_{t}\right): x_{t+1}\right)\right. \\
& \left.\quad \neq\left(I^{n+t-1}, x, x_{2}, \ldots, x_{t}\right)\right\}-1,
\end{aligned}
$$

where $x_{2}, \ldots, x_{t+1}$ is any superficial sequence for $I /(x)$. This completes the proof of the theorem. 
This theorem gives necessary conditions for a graded ring $S=S_{0}\left[S_{1}\right]$ to be the associated graded ring of a CM local ring. For example, consider the graded ring $S=k[x, y] /\left(x^{2}, x y\right)$ where $k$ is any field. Then

$$
H_{S^{+}}^{0}(S) \cong x S \text { and } H_{S^{+}}^{1}(S) \cong y^{-1} T,
$$

where $T=k\left[y^{-1}\right]$. Therefore $a_{0}(S)=1$ and $a_{1}(S)=-1$, so $S$ is not the associated graded ring of a 0 -dimensional ideal in a $\mathrm{CM}$ local ring.

As an immediate corollary, we recover the following result (cf. [S, Proposition 3; M, Theorem 2]).

Corollary 2.2. Let $(R, m)$ be a $C M$ local ring of dimension $d$ and $I$ an $m$ primary ideal. Suppose grade $g r_{I}(R)^{+} \geq d-1$. Then for all minimal reductions $J$ of $I$

$$
r_{J}(I)=n(I)+d=a_{d}(I)+d .
$$

Proof. Combine part (a) of Theorem 2.1 with part (c) of Remark 1.4.

\section{ApPlications IN DIMENSION TWO}

As an application of Theorem 2.1, we examine the independence of the reduction number of $m$-primary ideals in two-dimensional $C M$ local rings. By virtue of Trung and Huckaba's result, the interesting case is when grade $g r_{I}(R)^{+}=0$. In this case, we know $a_{0}(I)<a_{1}(I)$. If $a_{1}(I)<a_{2}(I)$ then by part (c) of Remark $1.4 r_{J}(I)=n(I)+2$ for all $J \in M R(I)$. So if the reduction number depends on the choice of minimal reduction, we must have $a_{1}(I) \geq a_{2}(I)$, in which case part (b) of Theorem 2.1 can be applied.

Proposition 3.1. Let $(R, m)$ be a two-dimensional $C M$ local ring and $I$ an $m$ primary ideal. Suppose $P_{I}(n)=H_{I}(n)$ for $n \geq 2$. Then $r_{J}(I)=r(I)$ for all $J \in M R(I)$.

Proof. As mentioned above, if grade $g r_{I}(R)^{+}>1$, we are done. So suppose grade $g r_{I}(R)^{+}=0$. By Theorem 2.1 we have $a_{0}(I)<a_{1}(I)$. If $a_{1}(I) \leq a_{2}(I)+$ 1 , then we are done by Lemma 1.2. On the other hand, if $a_{1}(I)>a_{2}(I)+1$ then $a_{1}(I)=n(I)$ by part (b) of Remark 1.4. Since $n(I) \leq 1$, Lemma 1.2 yields that $r_{J}(I) \leq 2$ for all $J \in M R(I)$. But if $r_{J}(I) \leq 1$ then $g r_{I}(R)$ is CM (see [V]), a contradiction. Therefore, if $a_{1}(I)>a_{2}(I)+1$ then $r_{J}(I)=2$ for all $J \in M R(I)$.

This argument can be pushed further in the case $I$ is the maximal ideal:

Remark 3.2. Let $(R, m)$ be a two-dimensional CM local ring and suppose that $P_{m}(n)=H_{m}(n)$ for $n \geq 3$. Then $r_{J}(m)=r(m)$ for all $J \in M R(m)$.

Proof. As in the proof of Proposition 3.2, we may assume grade $g r_{m}(R)^{+}=0$ and $a_{1}(m)>a_{2}(m)+1$. Hence $a_{1}(m)=n(m) \leq 2$ and so $r_{J}(m) \leq 3$ for all $J \in M R(m)$. We claim that $r_{J}(m)=3$ for all $J \in M R(m)$. Suppose this is not the case for some $J \in M R(m)$. Since $r_{J}(m) \leq 2$ we have that $m^{n} \cap J=J m^{n-1}$ for $n \geq 3$. But $m^{2} \cap J=J m$ since any minimal generating set for $J$ forms part of a minimal generating set for $m$. By Proposition 2.6 of [VV], $g r_{m}(R)$ is CM, a contradiction.

Proposition 3.3. Let $(R, m)$ be a two-dimensional $C M$ local ring and $I$ an $m$ primary ideal. Suppose there exists an element $x \in I \backslash I^{2}$ such that $\left(I^{m}: x\right)=$ $I^{m-1}$ for $m \geq 3$. Then $r_{J}(I)=r(I)$ for all $J \in M R(I)$. 
Proof. As before, assume grade $g r_{I}(R)^{+}=0$. By part (a) of Theorem 2.1, $a_{0}(I)<a_{1}(I)$. If $a_{1}(I)<a_{2}(I)$, we are done by Lemma 1.2. If not, then by part (b) of Theorem 2.1,

$$
a_{1}(I)=\max \left\{n \in \mathbf{Z} \mid\left(I^{n+1}: x\right) \neq I^{n}\right\} \leq 1 .
$$

This implies (as in the proof of Proposition 3.1) that $r_{J}(I)=2$ for all $J \in$ $M R(I)$.

The following example, computed with the help of D. Bayer and M. Stillman's "Macaulay" program, illustrates that Propositions 3.1 and 3.3 are in some sense the best possible.

Example 3.3. Let $R=k[x, y]_{(x, y)}$ and $I=\left(x^{7}, x^{6} y, x^{3} y^{4}, x^{2} y^{5}, y^{7}\right)$. Then we find that $n(I)=2$ and $\left(I^{m}: y^{7}\right)=I^{m-1}$ for $m \geq 4$. However, if $J_{1}=$ $\left(x^{7}, y^{7}\right)$ and $J_{2}=\left(x^{7}, x^{6} y+y^{7}\right)$, then $r_{J_{1}}(I)=3$ while $r_{J_{2}}(I)=2$.

\section{ACKNOWLEDGMENT}

I would like to thank C. Huneke and B. Johnston for many stimulating discussions regarding this material. I am also grateful to the referee for pointing out Remark 3.2.

\section{REFERENCES}

[EGA] A. Grothendieck and J. Dieudonné, Eléments de géométrie algébrique. III, Inst. Hautes Etudes Sci. Publ. Math. 11 (1961).

[H] S. Huckaba, Reduction numbers for ideals of higher analytic spread, Proc. Cambridge Philos. Soc. 102 (1987), 49-57.

[HM] S. Huckaba and T. Marley, Depth properties of Rees algebras and associated graded rings, J. Algebra (to appear).

[K] K. Kubota, On the Hilbert-Samuel function, Tokyo J. Math. 8 (1985), 439-448.

[M] T. Marley, The coefficients of the Hilbert polynomial and the reduction number of an ideal, J. London Math. Soc. (2) 40 (1989), 1-8.

[NR] D. G. Northcott and D. Rees, Reduction of ideals in local rings, Proc. Cambridge Philos. Soc. 50 (1954), 145-158.

[O] A. Ooishi, Genera and arithmetic genera of commutative rings, Hiroshima Math. J. 17 (1987).

[S] J. Sally, Reductions, local cohomology and Hilbert functions of local rings, Commutative Algebra (Durham 1981), London Math. Soc. Lecture Notes Ser., vol. 72, Cambridge Univ. Press, Cambridge, 1982, pp. 231-241.

[T] N. V. Trung, Reduction exponent and degree bound for the defining equations of graded rings, Proc. Amer. Math. Soc. 101 (1987), 229-236.

[V] G. Valla, On form rings which are Cohen-Macaulay, J. Algebra 58 (1979), 247-250.

[VV] P. Valabrega and G. Valla, Form rings and regular sequences, Nagoya Math. J. 72 (1978), 93-101.

[ZS] A. Zariski and P. Samuel, Commutative algebra, Vol. II, Van Nostrand, New York, 1960.

Department of Mathematics and Statistics, University of Nebraska, Lincoln, NEBRASKA 68588-0323

E-mail address: marley@hoss.unl.edu 